\section{Routine to provide a random order for counterbalanced variables}

\section{JAMES SCHEIRER}

State University of New York, Binghamton, New York 13901

One of the most common exercises in psychology is the randomization of a set of counterbalanced variables. While the problem is a logically simple one, the task itself can be laborious and time consuming. The routine described here accepts up to five variables, produces all combinations of levels of these variables, and prints a random order of this counterbalancing. After initialization, dimensioning and data input, the program is only 27 code statements long.

Table 1

Program to Provide Random Orders: Five Variables

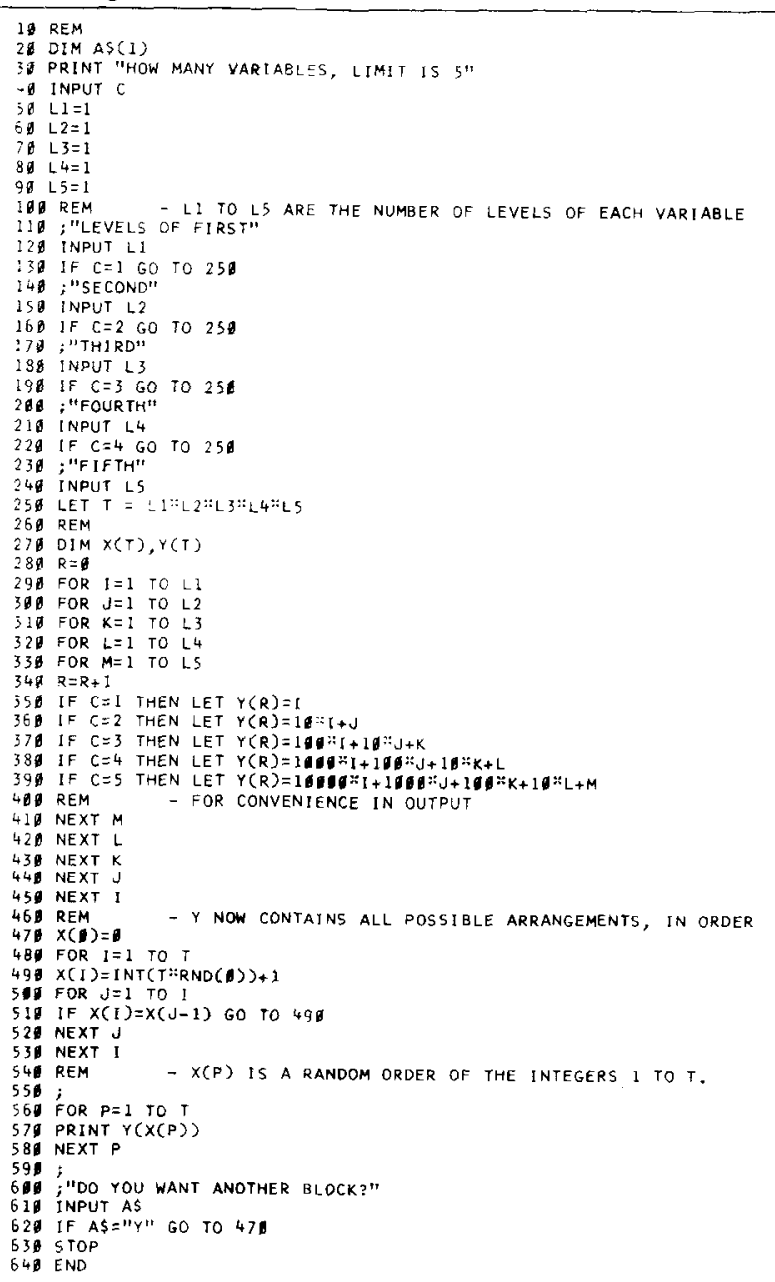

The program produces a systematic arrangement of all combinations of levels of variables and stores it in a Vector $Y$. It then randomly arranges the integers from 1 to the length of $Y(R)$ and stores each integer as a value of $X(P)$. The statement "PRINT $Y(X(P))$ " then produces the desired output.
It might be noted that the random ordering of a sequence for generation of a set of random sequences) of a specified length is a fairly common problem. In this program, this is accomplished in a subroutine of only seven code statements $(470-530)$ and is easily adaptable as a stand-alone program. In fact, if the user specifies only one variable with $\mathrm{L} 1$ levels, the output of this program is a random order of the sequence 1 to $\mathrm{L} 1$.

Support. This program was developed on an Interdata Model 70 minicomputer. It is written in INTERACTIVE BASIC but would be relatively simple to convert to BASIC or FORTRAN. With the Interdata Model 70 machine, the program uses 1,080 bytes of core before dimensioning.

Availability. Requests for copies of the program should be sent to C. James Scheirer, Department of Psychology, State University of New York at Binghamton, Binghamton, New York 13901.

\section{A BASIC subroutine to generate random Latin squares}

\section{JAMES SCHEIRER \\ State University of New York at Binghamton Binghamton, New York 13901}

Latin squares are useful in providing experimental arrangements for certain types of experimental designs. Tables exist for small squares from which it is possible to generate randomly all possible

Table 1 Generation of a Set of Random Latin Squares

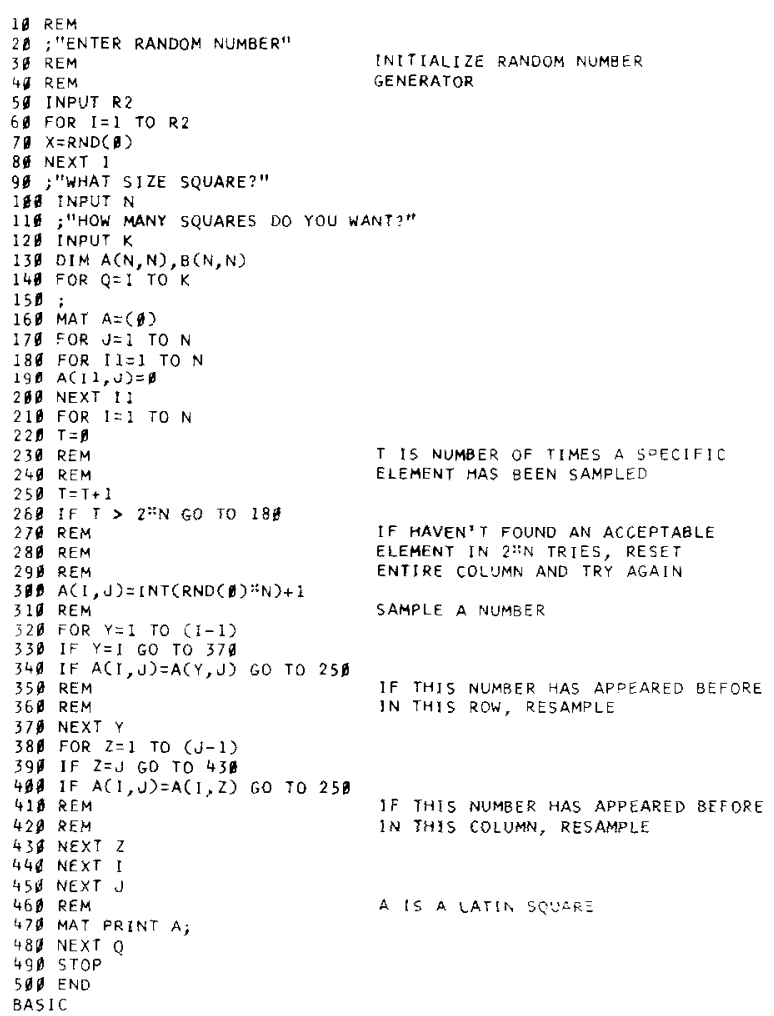


squares (ef. Fisher \& Yares. 1953). For 5 by 5 . or larger squares, hovever. there exists a large number of independent kernal squares. and tables allowing the generation of random squares are not conveniently available. The routine described here generates random Latin squares of any size.

For $a n n$ by $n$ square. this routine selects a number between 1 and $n$ at random. This number is then checked to see if it is permissible by comparing it to the other elements in its column and the other elements in its row. If the item is not permissible, a new number in the range $1-n$ is randomly selected. If $2 n$ selections do not find a permissible element (this will occur with probability $[(n-1) / n]^{2 n}$ in the worst case if such an element exists) all elements in the column are reset to zero and a new column is sampled. This escape is necessary since a square such as

$\left[\begin{array}{lll}1 & 2 & 0 \\ 2 & 1 & 0 \\ 3 & 0 & 0\end{array}\right]$

could occur by the procedures used. Obviously no permissible element exists for $A(3,2)$.

Support. This routine was developed on an Interdata Model 70 minicomputer. It is written in INTERACTIVE BASIC but would be easy to convert to BASIC or FORTRAN. On this machine, it uses 1.312 bytes of core before dimensioning.

Availability. Copies of th is program are available at no cost from C. James Scheirer, Department of Psychology. State University of New York at Binghamton. Binghamton. New York 13901.

\section{REFERENCE}

Fisher, R. A.. \& Yates. F. Statistical tables for biological. agricultural and medical research. Edinburgh: Oliver and Boyd. 1953. 